\title{
A Retrospective Review of Copy Number Variants and Ultrasound-Detected Soft Markers
}

\author{
Kajal Angras ${ }^{1 *}$, Lindsay A. Bailey ${ }^{1}$, Pallvi K. Singh ${ }^{1}$, Amanda J. Young ${ }^{2}$ and John Ross ${ }^{1}$ \\ ${ }^{1}$ Women's Health Service Line, Division of Maternal-Fetal Medicine, Geisinger, 100 N. Academy Avenue, Danville, PA 17822, USA \\ ${ }^{2}$ Biomedical and Translational Informatics Institute, Geisinger, 100 N. Academy Avenue, Danville, PA 17822, USA
}

\begin{abstract}
Objective: To examine the association of copy number variants (CNV) among fetuses with ultrasound-detected soft markers (USM).

Methods: This IRB-approved retrospective cohort study of fetuses with prenatal or children with postnatal chromosomal microarray analysis (CMA) sought to examine an association between clinically relevant CNV (classified as pathogenic CNV or variants of uncertain significance (VUS)) and USM in women who delivered at Geisinger between January 2010 and July 2018. The following USM were evaluated: choroid plexus cyst, thickened nuchal fold, absent or hypoplastic nasal bone, echogenic intracardiac focus, echogenic bowel, short long bones, and urinary tract dilation. Fetuses or children with known aneuploidy or a single gene disorder were excluded. Odds ratios (OR) of the association between CNV and USM were reported along with associated $95 \%$ confidence intervals $(\mathrm{Cl})$ and $\mathrm{p}$-values. P values $<0.05$ were considered significant.

Results: Ofthe 348 fetuses/children, 89 (25.6\%) hada clinically relevant CNV. Similar percentages of demographic, delivery and neonate characteristics were noted for those with a clinically relevant CNV and those with a normal microarray analysis. No statistically significant differences were noted am ong those fetuses/children with a clinically relevant CNV and structural anomaly ( $p=0.52 ;$ OR $1.18,95 \% \mathrm{Cl} 0.72-1.92)$, presence of one USM ( $p=0.72 ;$ OR 1.52, 95\% Cl 0.79-2.92), or presence of more than one USM ( $p=0.79 ;$ OR 1.56 , $95 \%$ Cl 0.28-8.72).
\end{abstract}

Conclusion: Our data supports a lack of association between a clinically relevant copy number variant and an ultrasound-detected soft marker. A small statistically insignificant increase in odds of a clinically relevant CNV was noted for those fetuses/children with one or more USM.

Keywords: Soft marker $\bullet$ Copy number variant $\bullet$ Microarray $\bullet$ Prenatal genetics

\section{Introduction}

Ultrasound-detected soft makers (USM) are second trimester sonographic findings that increase the suspicion for aneuploidy in the fetus. Softmarkers may be present in $11-17 \%$ of normal pregnancies without structural abnormalities or aneuploidy [1]. Genetic screening or testing (typically in the form of cell-free fetal DNA screening test or an amniocentesis) is offered to high-risk patients with USM to evaluate for aneuploidy. When isolated or in the absence of anomalies, soft markers do not significantly increase the risk of aneuploidy and often do not require further follow-up or evaluation [2]. Previous studies have been primarily based on the association between USM and chromosomal alterations determined by traditional karyotyping which cannot detect alterations less than five megabases, on average.

Chromosomal microarray analysis (CMA) offers the advantage of detecting duplications or deletions assmall as 50 to 100 kilobases that would have been otherwise missed on traditional karyotyping [3]. Recent evidence has favored CMA over conventional karyotyping to detect the presence of clinically relevant copy number variants (CNV) in the setting of fetal ultrasound anomalies [4]. Clinically relevant CNV are defined as submicroscopic deletions or duplications of genetic material identified on CMA and classified as either pathogenic or variants of uncertain significance (VUS). Clinically relevant CNV may be detected in $6-10 \%$ of anomalous fetuses with a normal karyotype and $2 \%$ of non-anomalous fetuses with a normal karyotype $[5,6]$. Because the American College of Obstetricians and Gynecologists (ACOG) and the Society of Maternal-Fetal Medicine (SMFM) support the use of CMA as a firsttier genetic test in women undergoing prenatal diagnostic testing, it is rapidly

*Address for Correspondence: Dr. Kajal Angras, Women's Health Service Line, Division of Maternal-Fetal Medicine, Geisinger, 100 N. Academy Avenue, Danville, PA 17822, USA, Tel: (570) 271-8160; E-mail: kangras@geisinger.edu

Copyright: (c) 2020 Angras $K$, et al. This is an open-access article distributed under the terms of the Creative Commons Attribution License, which permits unrestricted use, distribution, and reproduction in any medium, provided the original author and source are credited.

Received 14 April 2020; Accepted 28 April 2020; Published 05 May 2020 becoming the recommended test, especially in the setting of a fetal anomaly $[3,7]$.

There is a paucity of literature to demonstrate an association between CMA aberration and USM. In the setting of an identified USM, there is a lack of standardization in performing genetic screening or diagnostic testing, which may contribute to feelings of anxiety and confusion in expecting parents. The lack of literature and inconsistency of care make it difficult to counsel patients on the potential association between USM and chromosomal abnormalities. Evidence to use CMA for identifying an underlying genetic aberration in a fetus with USM is lacking. Studies evaluating the presence of CMA with structural anomalies excluded soft markers in their analysis [8]. The primary aim of our study was to evaluate the association of clinically relevant CNV among fetuses with one or more USM, with or without a structural abnormality. We hypothesized that there would be an increased risk of clinically relevant CNV among fetuses with one or moreUSM.

\section{Materials and Methods}

Weconductedan institutional review board-approved retrospectivecohort study of singleton pregnancies who delivered at Geisinger between January 2010 and July 2018. A search of electronic medical records was performed using International Classification of Diseases (ICD) codes. Pregnant women who underwent an amniocentesis or children who underwent postnatal genetic evaluation with CMA (if prenatal testing was not performed) and had antenatal ultrasound performed between 13 weeks and 32 weeks gestation at Geisinger were included in the study. Fetuses or children with known aneuploidy or single gene disorder were excluded. 379 patients who delivered at Geisinger during the study period met the inclusion criteria.

Charts of all of the study patients were reviewed by the study investigators (KA, LB, PS) to abstract the baseline characteristics and CMA results. Ultrasound reports for all of the study patients were also reviewed. The following ultrasound-detected soft markers were evaluated: choroid plexus cyst, thickened nuchal fold (defined as $\geq 6 \mathrm{~mm}$ before 21 weeks gestation), hypoplastic or absent nasal bone, echogenic intracardiac focus, echogenic bowel, shortlong bones (defined as $\leq 3$ standard deviation below the mean), 
and urinary tract dilation (defined as anterior-posterior diameter $\geq 4 \mathrm{~mm}$ before 28 weeks gestation or $\geq 7 \mathrm{~mm}$ at 28 weeks gestation or greater).

For CMA results that were reported prenatally, amniotic fluid samples were obtained by means of amniocentesis. Indications for prenatal amniocentesis including the following: advanced maternal age, structural abnormality, abnormal prenatal genetic screening, or maternal anxiety. For cases in which an amniocentesis was offered but not performed, CMA was performed on postnatal samples obtained from either neonatal or pediatric blood collection. Postnatal CMA results were included in our analysis as it is routinely performed in the pediatric population in the setting of prenatally detected anomalies, developmental delays, or seizure disorder. Two clinical genetic testing laboratories performed the CMA. One of the laboratories used a single nucleotide polymorphism (SNP) based CMA platform, while theother used a SNP based platform in conjunction with array comparative genome hybridization (array CGH). Both laboratories were CLIA certified and were used routinely in prenatal and pediatric genetics clinics within Geisinger for microarray analysis during the study period. CMA testing designated CNV as pathogenic (abnormal), variants of uncertain significance (VUS), or benign. Pathogenic variants were defined as deletions or duplications that were not found in population databases and were suspected to be disease causing based on the CNV size and gene content. VUS results were classified as such if there was inadequate information about the deletion or duplication, but the CNV could not be categorized as benign as it was not present in population databases. Benign variants were CNV that were found in the general population in healthy individuals and were not expected to cause disease. Benign CNV or common population variants were not included in the analysis. The categories of variant classification are standard reporting nomenclature, and the classification of these variants was not altered from the laboratory analysis.

Children with structural anomalies were classified by the anatomic system for which an abnormality was present: central nervous system, craniofacial, cardiac, thorax, musculoskeletal, gastrointestinal tract, and genitourinary tract. Data were summarized by frequencies and percentages for categorical variables. Mean and standard deviation (for normal distribution) or median and interquartile range (for non-normal distribution) was reported for continuous variables. Comparison of the baseline characteristics in the two cohorts (CNV positive and CNV negative) was performed using Chi-squared tests. Odds ratios (OR) with respective $95 \%$ confidence intervals $(\mathrm{Cl})$ and Chi-square test statistics were reported from a univariate logisticregression model. P values $<0.05$ were considered significant. All statistical analysis was performed using SAS v 9.4 (SAS Institute, Inc., Cary, NC).

\section{Results}

Of the 379 women that met our inclusion criteria, 31 were excluded due to a known singlegene disorder or aneuploidy resulting in a total of 348 women for analysis; $25.6 \%$ (89/348) of which had a fetus/child with a clinically relevant CNV (CNV +) and 74.4\% (259/348) with a normal microarray analysis (CNV-).
The majority of the CMA results were obtained on postnatal testing. CNV - and $\mathrm{CNV}+$ groups were similar with respect to maternal age at delivery, maternal race, tobacco use, the mode of delivery, the gestational age at delivery, the gender of the child, admission to the neonatal intensive care unit(NICU), and the presence of structural anomaly (Tables 1 and 2). When examining the total cohort (CNV + and CNV -), 89.6\% (312/348) of the women were noted to be less than 35 years of age, $91.9 \%$ (320/348) were non-Hispanic white, and $77.0 \%$ (268/348) werenon-smokers. The majority of the patients, $39.4 \%$ $(137 / 348)$ had a female neonate and $69.8 \%(238 / 348)$ delivered at term or later (Table 1).

Out of the total cohort, $84.2 \%$ (293/348) of the children had a normal ultrasound and $15.8 \%$ (55/348) of the children had presence of one or more USM (Table 3). In the group withoutan identified USM, 24.2\% (71/293) of the cases were CNV +, while the remaining $75.8 \%$ (222/293) of cases were CNV Of the 55 cases with USM, $67.3 \%$ (37/55) had negative testing for CNV while $32.7 \%(18 / 55)$ of cases were CNV +. Overall, no association was detected between a clinically relevant CNV and one or more USM. However, a small increased odds was noted, although not statistically significant, of detecting a clinically relevant CNV with one USM (OR 1.52,95\% CI 0.8-2.8) and with more than one USM (OR 1.56, 95\% Cl 0.3-8.7) (Table 2). Amongst the total cohort, urinary tract dilation and choroid plexus cyst were identified as the most common USM detected. Parental testing for the CNV was performed for the majority of the cases after a clinically relevant CNV was identified in the proband. Of note, most of the CMA aberrations were identified to be maternally or paternally inherited, rather than de novo.

In addition to individuals with an identified USM, the study assessed the association between identified structural abnormalities and clinically relevant CNV. In the CNV + group, 59.6\% (53/89) of children/fetuses were noted to have a structural anomaly, while $40.4 \%$ (36/89) did not have an identified structural anomaly. The incidence of a clinically relevant CNV in children/fetuses with a structural anomaly was not significantly higher when compared with children/ fetuses who were CNV - with a structural anomaly. The odds of a clinically relevant CNV was not increased when one or more structural anomalies were present (OR 1.2, 95\% $\mathrm{Cl}$ 0.7-1.9) (Table 3). Musculoskeletal defects and congenital heart defects were the most common structural anomalies in the cohort.

\section{Discussion}

By performing a review of the CMA and the prenatal ultrasound reports, we were able to demonstrate an insignificant increase in the odds of a clinically relevant CNV in the presence of one or more USM compared with a normal ultrasound. Additionally, our findings noted an incidence of $32 \%$ for fetuses/ children with clinically relevant CNV when one or more USM was detected. Guo et al reported a similar incidence in the Chinese population [9]. Wang et al. reported an incidence of $15.1 \%$ of pathogenic, likely pathogenic, and VUS CNV in fetuses with USM [10].

We included variants of uncertain significance in our classification of

Table 1. Demographics and pregnancy characteristics.

\begin{tabular}{|c|c|c|c|c|}
\hline Patient and Clinical Characteristics & CNV negative $(n=259)$ & CNV positive $(n=89)$ & Odds Ratio (95\% Cl) & $P$-value \\
\hline $\begin{array}{l}\text { Age at Delivery }(\%) \\
\leq 34 \text { years }\end{array}$ & $230(88.8)$ & $82(92.1)$ & $0.68(0.29-1.61)$ & 0.38 \\
\hline$>34$ years & $29(11.2)$ & $7(7.9)$ & & \\
\hline Race (\%): Caucasian & $237(91.5)$ & $83(93.3)$ & $0.78(0.31-1.99)$ & 0.61 \\
\hline Ethnicity (\%): Non-Hispanic & $235(90.7)$ & $85(96.6)$ & $0.34(0.10-1.16)$ & 0.08 \\
\hline Tobacco use (\%): Nonsmokers & $202(78)$ & $66(74.2)$ & $1.23(0.71-2.16)$ & 0.46 \\
\hline Mode of delivery (\%): Vaginal & $146(56)$ & $52(58)$ & $0.92(0.57-1.51)$ & 0.75 \\
\hline $\begin{array}{l}\text { Gestational age at delivery (\%): } \\
\geq 37 \text { weeks' gestation }\end{array}$ & $179(68.8)$ & $64(71.3)$ & $0.89(0.52-1.51)$ & 0.66 \\
\hline Gender of neonate (\%): Female & $105(40.5)$ & $32(36)$ & $1.21(0.74-2.00)$ & 0.45 \\
\hline NICU admission (\%): Yes & $140(54.1)$ & $41(46.1)$ & $0.73(0.45-1.18)$ & 0.19 \\
\hline
\end{tabular}


Table 2. Clinically relevant CNV and ultrasound-detected soft marker.

\begin{tabular}{|c|c|c|c|c|}
\hline Ultrasound-Detected Soft Marker & CNV negative ( $n=259$ ) & CNV positive $(n=89)$ & Odds Ratio (95\% Cl) & $P$-value \\
\hline Absent (\%) & $222(85.7)$ & $71(79.8)$ & & \\
\hline Present (\%) & $37(14.3)$ & $18(20.2)$ & $1.52(0.82-2.84)$ & 0.19 \\
\hline Ultrasound-Detected Soft Marker & CNV negative ( $n=259$ ) & CNV positive $(n=89)$ & Odds Ratio (95\% Cl) & $P$-value \\
\hline Absent (\%) & $222(85.7)$ & $71(79.8)$ & & \\
\hline 1 USM (\%) & $33(12.7)$ & $16(18)$ & $1.52(0.79-2.92)$ & 0.72 \\
\hline > 1 USM (\%) & $4(1.5)$ & $2(2.2)$ & $1.56(0.28-8.72)$ & 0.79 \\
\hline
\end{tabular}

Note: Cl: Confidence Interval; CNV: Copy Number Variants

Table 3. Clinically relevant CNV and structural anomaly.

\begin{tabular}{cccc}
\hline Structural Anomaly & CNV negative (n= 259) & CNV positive (n= 89) & Odds Ratio (95\% Cl) \\
\hline Absent (\%) & $115(44.4)$ & $36(40.4)$ & \\
\hline Present (\%) & $144(55.6)$ & $53(59.6)$ & $1.18(0.72-1.92)$
\end{tabular}

clinically relevant CNV. While the CMA results classified as VUS were not associated with a specific microdeletion or microduplication syndrome, their size and gene content kept them from a benign classification. As more genetic information becomes available, it is possible that CNV may change classification from VUS to benign. While the inclusion of VUS results contributed to our overall incidence of clinically relevant CNV in the setting of one of more USM detected, we felt it was important to take these into account to provide the most comprehensive assessment when evaluating for an association between clinically relevant CNV and USM.

One of the strengths of our study is that we are one of the few studies to examine the association between CMAaberration and USM in the United States (US). Wang et al. were among the first to examine the association between pathogenic CNV and USM [11]. However, the study was conducted in the Chinese population. Wang et al. performed the CMA and clinical interpretation on their subjects, and their clinical interpretation may be different than that of a United States CLIA certified clinical laboratory. Ginsberg et al. reported a high recurrence rate of a solitary USM implying a genetic predisposition [12]. It was therefore necessary for us to examine if the association existed for the US population. Previous studies have evaluated the relationship between CNV and an isolated USM such as increased nuchal translucency, hypoplastic nasal bone, ventriculomegaly, or echogenic bowel [13-16]. Tothe best of our knowledge, our study is one of the first studies to evaluate the relationship between CNV and one or more USM. Furthermore, our data was collected from a single tertiary center across an 8-year time span. The group of physicians and sonographers over the span of the study period followed department protocols that minimized variation in reporting practices. The standardized protocols allowed for consistency in performing and interpreting ultrasound for USM.

However, our study is not without limitations. Our cohort was made of a uniform population consisting of mainly young Caucasians, making it difficult to generalize our results to an ethnically diverse patient population. Although we noted an increased trend, our study was underpowered to detect a statistical significance which may exist if we had a larger sample size. Additionally, we were unable to analyze the presence of clinically relevant CNV by specific USM due to the small number of fetuses with one or more USM. Lastly, the majority of the CMA results in our study were on postnatal samples which potentially may lead to selection bias. It is important to recognize that postnatal CMA have a better resolution and interpretations than those used in a prenatal setting. ${ }^{7}$ This may identify a clinically relevant CNV that would not have been identified prenatally in some cases, which may lead to slight overestimation of the frequency of clinically relevant CNV in our study.

Understanding the association between USM and pathogenic or uncertain CNV, will allow health care providers to better counsel patients on the risk of a genetic etiology, when an USM is identified. In order to offer the most appropriate testing options and counsel patients on the potential causes, we need to further examine the association between an USM and CNV.
A larger, adequately powered study would need to be pursued to definitively confirm our study findings. Further studies on this topic may seek to understand the difference between the association of USM with both pathogenic CNV and uncertain CNV. With additional research and a larger study population, health care providers may be able to counsel patients and make recommendations in the setting of a prenatal USM.

\section{Conclusion}

In summary, we noted an increased odd, although small and insignificant, of having a clinically relevant CNV among fetuses with one or more USM. CMA allows clinicians to detect segmental duplications or deletions that may impact health and wellness of the fetus, and also provide an explanation for the USM. Further research is required to provide frequency estimates of copy number variants associated with ultrasound-detected soft markers to determine if invasive testing with chromosomal microarray should be incorporated into the discussion and counseling in the setting of an identified ultrasound soft marker.

\section{Funding}

The research was supported and funded by Geisinger Clinic.

\section{Conflict of Interest}

The authors report no conflicts ofinterest.

\section{References}

1. Breathnach, Fionnuala $M$, Ann Fleming, and Fergal D Malone. "The Second Trimester Genetic Sonogram". Am J Med Genet C Semin Med Genet 145 (2007): 62-72.

2. Norton, Mary E. "Follow-up of sonographically detected soft markers for fetal aneuploidy". Semin Perinatol 37 (2013): 365-369.

3. Committee on Genetics and the Society for Maternal-Fetal Medicine. "Microarrays and Next-Generation Sequencing Technology: The Use of Advanced Genetic Diagnostic Tools in Obstetrics and Gynecology". Obstet Gynecol 128 (2016): e262-e268.

4. De Wit MC, Srebniak, LCP Govaerts and Diane Van Opstal, et al. "Additional Value of Prenatal Genomic Array Testing in Fetuses with Isolated Structural Ultrasound Abnormalities and A Normal Karyotype: A Systematic Review of The Literature". Ultrasound Obstet Gynecol 43 (2014):139-146.

5. Callaway, Jonathan LA, Lisa G. Shaffer, Lyn S. Chitty and Jill A. Rosenfeld, etal." The Clinical Utility of Microarray Technologies Applied to Prenatal Cytogenetics in the Presence of a Normal Conventional Karyotype: A Review of The Literature". Prenat Diagn 33 (2013): 1119-1123. 
6. Wapner, Ronald J, Christa Lese Martin, Brynn Levy and Blake C. Ballif, et al. "Chromosomal Microarray versus Karyotyping for Prenatal Diagnosis". N Engl J Med 367 (2012): 2175-2184.

7. Dugoff, Lorraine, Mary E. Norton, Jeffrey A. Kuller and Society for Maternal-Fetal Medicine. "The Use of Chromosomal Microarray for Prenatal Diagnosis". Am J Obstet Gynecol 215 (2016): B2-9.

8. Donnelly, Jennifer C, Lawrence D Platt, Andrei Rebarber and Julia Zachary. "Association of Copy Number Variants with Specific Ultrasonographically Detected Fetal Anomalies". Obstet Gynecol 124 (2014): 83-90.

9. Guo YL, L Wang, SW Xue and SZQu, et al. “Application of Single Nucleotide Polymorphism Array in Prenatal Diagnosis for Fetuses with Abnormal Ultrasound Findings". Zhonghua Fu Chan Ke Za Zhi 53 (2018): 464-470.

10. Wang, Jing, Lin Chen, Cong Zhou and Li Wang, et al. "Prospective Chromosome Analysis of 3429 Amniocentesis Samples in China Using Copy Number Variation Sequencing”. Am J Obstet Gynecol 219 (2018): 287.e1-287.e18.

11. Wang, Jing, Lin Chen, Cong Zhou and LiWang, etal. “Identification of Copy Number
Variations among Fetuses with Ultrasound Soft Markers Using Next-Generation Sequencing". Sci Rep 8 (2018): 1-7.

12. Ginsberg, Yuval, Nizar Khatib, Zeev Weiner and Ron Beloosesky, et al."The Recurrence of Sonographic 'Soft Markers': Ominous Sign or 'Just' Genetics?" Prenat Diagn 37 (2017): 469-472.

13. Grande M, FAR Jansen, YJ Blumenfeld and A Fisher, et al. "Genomic Microarray in Fetuses with Increased Nuchal Translucency and Normal Karyotype: A Systematic Review and Meta-Analysis". Ultrasound Obstet Gynecol 46 (2015): 650-658.

14. Gu, Ying Zhi, Deborah L Nisbet, Karen L. Reidy, and Ricardo Palma-Dias. "Hypoplastic Nasal Bone: A Potential Marker for Facial Dysmorphism Associated with Pathogenic Copy Number Variants on Microarray". Prenat Diagn 39 (2019): 116-123.

15. Hu, P, Y Wang, R Sun and L Cao, et al. “Copy Number Variations with Isolated Fetal Ventriculomegaly”. Curr Mol Med 17 (2017): 133-139.

16. Singer, Amihood, Idit Maya, Arie Koifman and Nadra Nasser Samra, et al "Microarray Analysis in Pregnancies with Isolated Echogenic Bowel". Early Hum Dev 119 (2018): 25-28.

How to cite this article: Kajal Angras, Lindsay A. Bailey, Pallvi K. Singh, Amanda J. Young and John Ross. "A Retrospective Review of Copy Number Variants and Ultrasound-Detected Soft Markers." J Mol Genet Med 14 (2020): 448 doi: $10.37421 /$ jmgm.2020.14.448 